\title{
Facial expressions of emotion (KDEF): Identification under different display-duration conditions
}

\author{
Manuel G. Calvo \\ University of La Laguna, Tenerife, Spain \\ AND \\ DaNiel LuNDQvist \\ Karolinska Institutet, Stockholm, Sweden
}

\begin{abstract}
Participants judged whịch of seven facial expressions (neutrality, happiness, anger, sadness, surprise, fear, and disgust) were displayed by a set of 280 faces corresponding to 20 female and 20 male models of the Karolinska Directed Emotional Faces database (Lundqvist, Flykt, \& Ŏhman, 1998). Each face was presented under free-viewing conditions (to 63 participants) and also for $25,50,100,250$, and 500 msec (to 160 participants), to examine identification thresholds. Measures of identification accuracy, types of errors, and reaction times were obtained for each expression. In general, happy faces were identified more accurately, earlier, and faster than other faces, whereas judgments of fearful faces were the least accurate, the latest, and the slowest. Norms for each face and expression regarding level of identification accuracy, errors, and reaction times may be downloaded from www.psychonomic.org/archive/.
\end{abstract}

Faces and facial expressions of emotion are special types of stimuli, with high social and biological relevance. Accurate recognition of faces and their expressions allows a person not only to identify other people, but also to understand their states, needs, and intentions. This enables the observer to behave in a way that optimizes (or avoids) social interaction. There is evidence of automatic perception of affective facial expressions (Stenberg, Wiking, \& Dahl, 1998), with low perceptual thresholds and wide attentional span (Calvo \& Esteves, 2005). Extensive reviews and thorough discussions about the reduced processing requirements, or enhanced sensory processing, of affective facial expressions, and the neural mechanisms involved, have been provided recently by Compton (2003), Pessoa (2005), Palermo and Rhodes (2007), and Vuilleumier and Pourtois (2007). This special cognitive capacity for facial processing is supported by neural mechanisms that specialize in the recognition of faces (Farah, 2000) and by neural mechanisms that are especially sensitive to early detection and discrimination of emotional expressions (see, e.g., Morris, Öhman, \& Dolan, 1998; Schupp et al., 2004).

Given the adaptive importance of affective facial expressions, it is understandable that considerable behavioral and neuropsychological research has been devoted to the study of emotional face processing (see Adolphs, 2002). Standardized sets of emotional faces have been used by researchers, with the Pictures of Facial Affect (PFA-Ekman
\& Friesen, 1976) used in the majority of studies. Although very valuable, this set of pictures contains photographs of only five males and six females, displaying each of the six basic facial expressions (happiness, sadness, anger, fear, disgust, and surprise), in addition to neutral expressions. Researchers in this field would benefit from a larger sample of pictures. To this end, new databases have been offered more recently (Gur et al., 2002; Mazurski \& Bond, 1993; Tottenham, Borscheid, Ellertsen, Marcus, \& Nelson, 2002; Watson, 2001).

Palermo and Coltheart (2004) selected a sample of 336 faces from the five aforementioned face databases, with seven expressions displayed by 25 female and 25 male models. In a normative study, these authors presented the faces to 12 female and 12 male participants, who judged each expression as neutral, happy, angry, disgusted, fearful, sad, or surprised. Recognition rates and types of errors, as well as response times, were collected, thus producing norms about how accurately and quickly each expression of each model is identified. These data have provided researchers on the processing of affective facial expressions with a valuable tool that can be used for stimulus selection.

The purpose of the present study is to generate normative data for another face database, the Karolinska Directed Emotional Faces (KDEF-Lundqvist, Flykt, \& Ohman, 1998; see a detailed description in the Method section of Experiment 1). The KDEF has been recently used in 
a considerable number and a wide range of studies (see AppendixC.pdf in the Archived Materials). The KDEF is a large database from which we used 40 models (20 females and 20 males) displaying each of seven expressions. In comparison with Palermo and Coltheart (2004), we have made the following contributions. First, norms for a new set of stimuli are available for researchers. Second, we significantly increased the number of participants who judged the facial expressions (63 in Experiment 1 and 160 in Experiment 2), thus trying to obtain relatively stable and generalizable data. Third, in addition to examining accuracy, errors, and response times for each stimulus under free-viewing conditions (Experiment 1), as Palermo and Coltheart did, we presented the face stimuli under each of five fixed-exposure conditions $(25,50,100,250$, and $500 \mathrm{msec}$ ). This is important for determining the perceptual threshold levels for each stimulus. This information should be particularly useful for selecting stimuli in studies on the automatic nature and the time course of emotional face processing.

\section{EXPERIMENT 1 Free-Viewing Conditions}

\section{Method}

Participants. Thirty-two females (mean age, 22.5 years, $S D=$ 3.2) and 31 males (mean age, 22.7 years, $S D=3.4$ ) participated for course credit at La Laguna University.

Stimuli. KDEF stimuli were used. The KDEF is a set of 4,900 pictures of human facial expressions, created at Karolinska Institutet, Stockholm (for more details, see www.facialstimuli.com). The set contains pictures of 70 individuals ( 35 females and $\mathbf{3 5}$ males) displaying seven different emotional expressions (neutral, happy, angry, sad, disgusted, surprised, and fearful). Each expression is viewed from five different angles (full left profile, half left profile, straight, half right profile, and full right profile) and was photographed in two sessions. The pictures are color photographs. The models were Caucasian amateur actors (mean age, 25 years; range, 20-30 years). They were instructed to pose with the different expressions.

Out of the large KDEF database (70 models $\times 7$ expressions $X$ 5 perspectives), a final sample of 280 face stimuli were selected. This sample corresponded to 40 models ( 20 females and 20 males) with all seven expressions acceptable. All the selected face stimuli involved faces at a straight viewing angle. AppendixA.xls in the Archived Materials (first column) lists the original label for each of the selected pictures. We used various criteria for the selection of $\mathbf{4 0}$ models. First, for practical reasons, using all 70 models would have produced an unmanageable sample of 490 experimental stimuli (plus the practice trials, plus the training-and-test phase) for a given participant. Furthermore, we thought it was important that all faces be evaluated by the same participants, who thus served as their own controls, and so we discarded the possibility of two different samples of participants. Second, a few expressions of a few models were either damaged or over- or underexposed. This affects only single expressions of some models, and therefore all the other expressions can be used in studies that do not need to compare all seven expressions. In our case, such comparisons were essential, and so only those models with all expressions in good form could be used. We excluded 3 male and 2 female models for this reason. Finally, in a preliminary screening, we thought that some expressions of some models were not genuine or not totally compelling, in the sense that they did not very clearly depict the intended emotional expression. This implied that the models at issue were excluded for all expressions, even though most of these are valid and can be used separately. In general, if researchers want to use separate expressions from each model, the sample of valid KDEF stimuli can be significantly increased far beyond the current 40-model sample.

For the present study, the original KDEF stimuli were cropped using Adobe Photoshop 6.0 so that each face fit within an oval window subtending $20 \times 15 \mathrm{~cm}$ on a 17 -in. monitor. At approximately $80-\mathrm{cm}$ viewing distance during the experiments, this results in $14.2^{\circ} \times 10.7^{\circ}$ of visual angle. The hair, neck, and surrounding parts were darkened to remove distracting, noisy aspects that were not informative of emotional expression. Only the facial aspects that could convey emotional expression (i.e., forehead, eyes, nose, cheeks, mouth, and chin) were visible inside the oval window (see also Williams, Moss, Bradshaw, \& Mattingley, 2005).

Procedure. Participants were presented with the face stimuli on a super-VGA 17-in. monitor connected to a Pentium IV computer. Stimulus presentation and data collection were controlled by the E-Prime experimental software (Schneider, Eschman, \& Zuccolotto, 2002). Participants were informed that they would be presented with faces displaying one of six emotions (happy, angry, sad, disgusted, surprised, and fearful) or a neutral expression. They should try to identify the expression of each face and respond as soon as possible by pressing one of seven keys on a standard computer keyboard. The seven keys used were the space bar (right thumb), "s" (left ring finger), " $d$ " (left middle finger), " $f$ " (left forefinger), " $j$ " (right forefinger), " $\mathrm{k}$ " (right middle finger), and "l" (right ring finger). Above each of these keys was a written label with the word for the corresponding facial expression. The neutral expression had to be responded to by pressing the space bar, whereas the keys for the other expressions were counterbalanced so that each expression was associated with a different key for 1/7 (i.e., 9) of the participants.

The experimental session was composed of three phases. First, prior to presentation of the face stimuli for assessment of the emotional expression, the participants underwent a training phase. The participants were asked to learn to associate motor responses with verbal labels of emotion by practicing finger movements on the corresponding keys. Second, in a test phase, after the 15-min training phase, words representing each of the facial expressions were presented on the screen and participants responded by using the corresponding keys. This served to ensure that the associations had been learned and were equivalent for the different combinations of expression and key (see the Results section). During this test phase, only the words (i.e., neutral, happy, angry, sad, disgusted, surprised, and fearful) defining the facial expressions were presented, rather than the photographs. Each word was randomly repeated nine times (three times in each of three blocks). The participants pressed the corresponding key for each word. Finally, in the experimental phase, the face stimuli were presented one at a time in the center of the screen. Participants judged which expression was displayed by the face, pressing the corresponding key. Following 14 practice trials (seven expressions each for 1 female and 1 male model), each participant was presented with 280 experimental trials (all seven expressions of each of the $\mathbf{4 0}$ models). Each face was shown only once to each participant. There were seven blocks of 40 trials each, with the order of blocks and trials randomized. The verbal labels remained the same on the keyboard during all three phases for each participant.

\section{Results}

Reaction times to words (test phase). The participants' reaction times for the words that represented each face expression were analyzed in a 3 (block) $\times 7$ (expression: neutral, happy, angry, sad, disgusted, surprised, or fearful) ANOVA with repeated measures. Main effects of block $\left[F(2,124)=119.19, M S_{\mathrm{e}}=54,518, p<\right.$ $.0001]$ and expression $\left[F(6,372)=9.16, M S_{\mathrm{e}}=75,621\right.$, $p<.0001]$ emerged. Reaction times decreased from the first $(M=1,153 \mathrm{msec})$ to the second $(M=1,012 \mathrm{msec})$ block and from the second to the third $(M=911 \mathrm{msec})$ 
block (all Bonferroni-corrected ps $<.0001$ ). In addition, response latencies were shorter for the neutral label $(M=$ $929 \mathrm{msec}$ ) than for the other labels (happy, $1,073 \mathrm{msec}$; angry, $1,079 \mathrm{msec}$; sad, $1,084 \mathrm{msec}$; disgusted, $977 \mathrm{msec}$; surprised, $989 \mathrm{msec}$; fearful, 1,045 msec). However, these effects were qualified by an interaction $[F(12,744)=$ 2.02, $\left.M S_{\mathrm{e}}=73,304, p<.025\right]$. As revealed by separate ANOVAs for each block, this interaction resulted from the fact that there was an effect of expression in both the first $\left[F(6,372)=8.54, M S_{\mathrm{e}}=54,685, p<.0001\right]$ and the second $\left[F(6,372)=6.30, M S_{\mathrm{e}}=50,816, p<.0001\right]$ block, but not in the third block $\left[F(6,372)=1.22, M S_{\mathrm{e}}=42,605, p=\right.$ .30, n.s.]. This shows that the motor responses to the different stimuli and for the different keyboard locations were comparable (neutral, $874 \mathrm{msec}$, happy, $938 \mathrm{msec}$; angry, 937 msec; sad, 922 msec; disgusted, 885 msec; surprised, $899 \mathrm{msec}$; fearful, $931 \mathrm{msec}$ ) at the end of the test phase.

Recognition rates of face expressions. The percentage of participants who judged each expression as the one intended by the model is shown in Figure 1 (see detailed information in AppendixA.xls in the Archived Materials). A three-way ANOVA was conducted on these proportion scores, with type of expression (neutral, happy, angry, sad, disgusted, surprised, or fearful) and face gender (female or male) as between-subjects factors. Gender of participant (female or male) was initially taken into account, but no differences emerged, and it was dropped from further analyses to reduce complexity. Significant effects of type of expression were examined with a posteriori Tukey HSD tests $(p<.05)$.There was a main effect of expression $\left[F(6,266)=17.08, M S_{e}=98.13, p<.0001\right]$, but not of face gender $(F<1)$. Neutral $(M=97.4 \%)$ and happy $(M=97.6 \%)$ expressions were identified more accurately than all the other expressions. Angry $(M=89.3 \%)$, sad $(M=87.2 \%)$, disgusted $(M=87.4 \%)$, and surprised $(M=$
86.2\%) expressions were identified more accurately than fearful $(M=79.3 \%)$ expressions. A borderline interaction $\left[F(6,266)=2.15, M S_{\mathrm{e}}=98.13, p=.05\right]$ reflected a tendency $[t(38)=1.96, p=.057]$ for angry expressions to be identified more accurately when posed by males $(M=$ $92.1 \%)$ than by females $(M=86.4 \%)$, whereas there was a tendency $[t(38)=1.83, p=.074]$ for disgusted expressions to be identified more accurately when posed by females $(M=91.1 \%)$ than by males $(M=83.6 \%)$.

Patterns of errors. The types of errors (i.e., the participants' responses that did not correspond to the intended expressions of the models) were also examined by means of a type of expression $\times$ face gender ANOVA. Figure 2 shows the proportion of participants labeling each type of expression as neutral, happy, angry, sad, disgusted, surprised, or fearful (see detailed information in AppendixA.xls in the Archived Materials). The effect of face gender was always $F<1$. All types of expressions were accurately classified to a significant extent (i.e., they were labeled as intended more likely than otherwise) [neutral, $F(6,228)=17,593.10$, $M S_{\mathrm{e}}=3.06$; happy, $F(6,228)=68,583.16, M S_{\mathrm{e}}=0.79$; angry, $F(6,228)=1,471.59, M S_{\mathrm{e}}=29.73$; sad, $F(6,228)=$ $1,270.31, M S_{\mathrm{e}}=32.75$; disgusted, $F(6,228)=695.54$, $M S_{\mathrm{e}}=60.18$; surprised, $F(6,228)=853.08, M S_{\mathrm{e}}=47.84$; fearful, $\left.F(6,228)=498.15, M S_{\mathrm{e}}=67.18\right]($ all $p s<.0001)$.

Bonferroni corrections $(p<.05)$ for multiple contrasts were conducted to examine differences between the types of errors. For neutral and for happy expressions, there were no significant differences between the various types of incorrect responses, all having a very low rate $(1 \%$ or less). Angry faces were misclassified most often as disgusted $(M=4.6 \%)$. This, however, was moderated by gender of model, as revealed by a type of expression $X$ face gender interaction $\left[F(6,228)=3.21, M S_{\mathrm{e}}=29.73\right.$, $p<.01]$, with female angry models being more likely

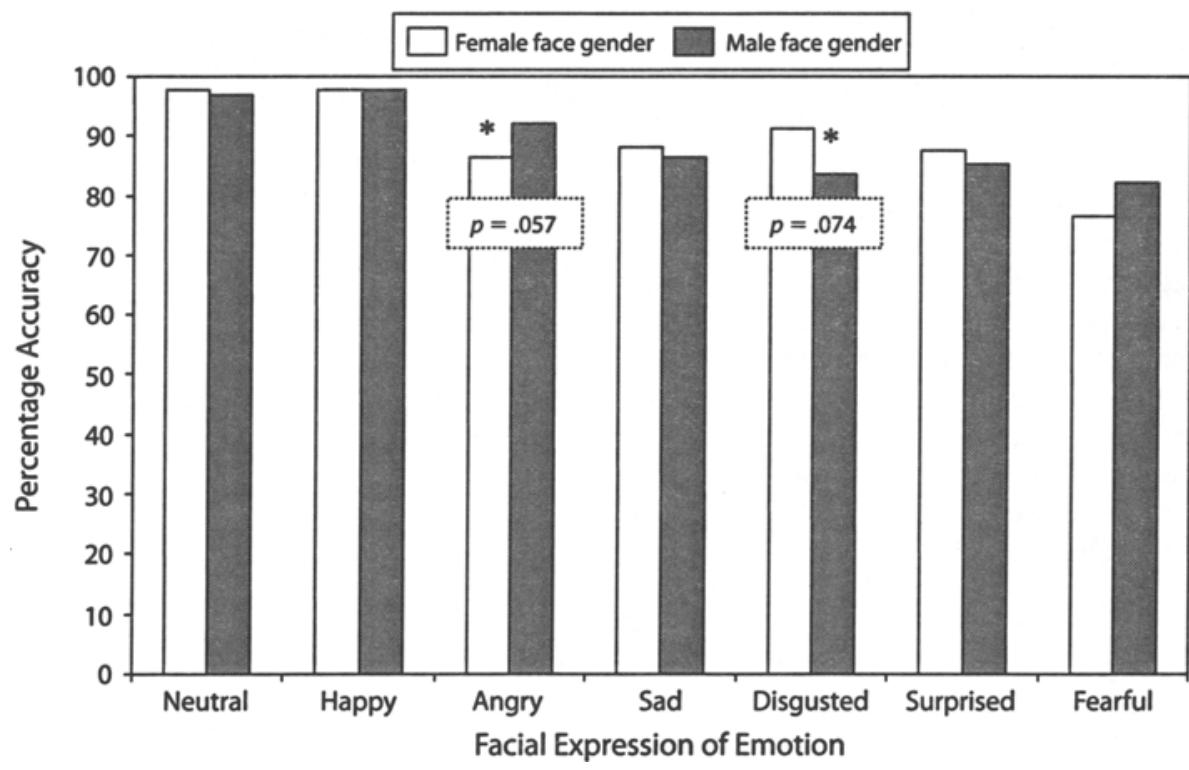

Figure 1. Percentage of expressions displayed, correctly judged to be the intended expression, as a function of female and male models. Asterisks indicate significant differences as a function of model gender. 


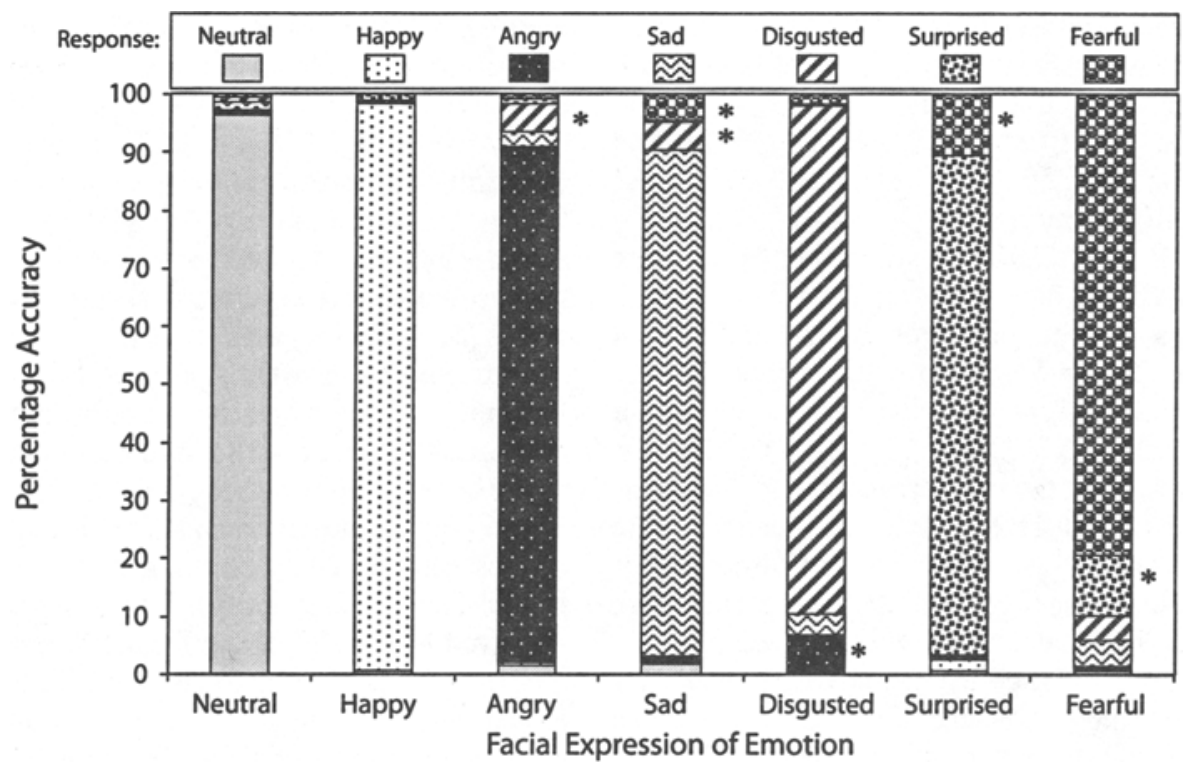

Figure 2. Percentage of responses of each type of expression for each facial expression category, indicating the types of errors made by the participants. Types of errors marked with an asterisk are significantly different from those not marked with an asterisk.

to be labeled as disgusted $(M=7.0 \%)$ than male angry models $(M=2.2 \%)$. Sad faces were misclassified most often as disgusted $(M=4.8 \%)$ or fearful $(M=4.6 \%)$. Disgusted faces were misclassified most often as angry $(M=6.6 \%)$. This, however, was moderated by gender of model, as revealed by a type of expression $\times$ face gender interaction $\left[F(6,228)=2.59, M S_{\mathrm{e}}=60.18, p<.025\right]$, with male disgusted models being more likely to be labeled as angry $(M=9.1 \%)$ than female disgusted models $(M=3.9 \%)$. Surprised faces were misclassified most often as fearful $(M=10.5 \%)$, whereas fearful faces were misclassified most often as surprised $(M=10.4 \%)$.

Response times. The average response times in the accurate classification of each of the seven facial expressions were also subjected to a type of expression (neutral, happy, angry, sad, disgusted, surprised, or fearful) $\times$ face gender (female vs. male) ANOVA. Incorrect responses (i.e., those in which the expression was judged to be different from the intended expression of the model) were not included in the analysis. RTs greater than $3,000 \mathrm{msec}$ and lower than 400 msec were removed as outliers ( $2.96 \%$ of the trials). The mean RTs for correctly recognizing each expression are shown in AppendixA.xls in the Archived Materials. There was a main effect of expression $[F(6,266)=99.41$, $\left.M S_{\mathrm{e}}=21,801.85, p<.01\right]$, with no significant effect of face gender $(F<1)$ or interaction $(F=1.32, p=.25)$. A Tukey HSD test for multiple contrasts revealed that happy $(M=1,091 \mathrm{msec})$ and neutral $(M=1,053 \mathrm{msec})$ expressions were correctly recognized more quickly than all the other expressions (all $p s<.001$ ), whereas fearful expressions were recognized significantly more slowly $(M=$ $1,715 \mathrm{msec} ;$ all $\mathrm{ps}<.001$ ). In addition, between these two extremes, surprise $(M=1,299 \mathrm{msec})$ was identified more quickly than anger $(M=1,444 \mathrm{msec})$ and sadness $(M=$ $1,508 \mathrm{msec}$; all $p \mathrm{~s}<.001$ ), which was identified more slowly than disgust $(M=1,361 \mathrm{msec} ; p<.001)$. There were no significant differences between the counterbalancing conditions in which the association between face stimulus expression and response key location was varied.

\section{EXPERIMENT 2 Fixed-Exposure Durations}

\section{Method}

Participants. One hundred sixty psychology undergraduates (125 females, 35 males; mean age, 22.2 years, $S D=3.1$ ) participated for course credit. They were selected from the same pool of undergraduate psychology students as those in Study 1 (all participants were different in each study). Given that there were no differences as a function of gender of participants in Study 1, and given the higher proportion of females than males, gender was not considered as a factor in Study 2. Nevertheless, the same proportion of participants of each sex ( 25 females and 7 males) was assigned to each of the five display time conditions of Study 2.

Stimuli and Procedure. In Study 2, the same KDEF pictures of facial expressions were used as in Study 1. A major difference in Study 2 involved the presentation of the face stimuli in a fixed-pace mode, so that each face was displayed during $25,50,100,250$, or $500 \mathrm{msec}$. The face stimuli were not backwardly masked by other pictorial stimuli. Rather, they were immediately replaced by large question marks in the center of the screen, which acted as a prompt for responding. Each participant saw all 280 experimental faces under the same fixed display condition. On each trial, a 500-msec cross in the center of the screen preceded the face stimulus, followed by a 4-sec interval in which white question marks were shown on a dark background screen. During this interval, the participant responded which expression was shown by the face by pressing a key, and then a new trial automatically started. Seven keys were associated with the seven verbal labels of expressions, in four different counterbalancing conditions (with eight participants in each condition, and each response key for a given expression being located in one of four different locations). Reaction times were not collected, for both theoretical and practical reasons. First, it was thought that in the fixed-pace display conditions of this experiment, accuracy 
would probably be more sensitive than reaction times. Second, a training-and-test-phase (as in Experiment 1) for associating keys and expressions would have been necessary to obtain reliable reaction times. However, this phase would have involved considerable additional time for a large sample of 160 participants, and so it was not conducted. The fact that there were no differences in accuracy between the four counterbalancing conditions confirms that key position did not affect response accuracy.

\section{Results}

The percentage of participants who judged each expression as the one intended by the model is shown in Figure 3 (see AppendixA.xls in the Archived Materials for detailed information). A three-way ANOVA was conducted on these proportion scores, with type of expression (neutral, happy, angry, sad, disgusted, surprised, or fearful), and face gender (female or male) as between-subjects factors and display time ( 25 vs. 50 vs. 100 vs. 250 vs. $500 \mathrm{msec}$ ) as a repeated measures factor. Significant effects of type of expression were examined with a posteriori Tukey HSD tests $(p<.05)$. Significant effects of display time were examined by means of Bonferroni-corrected a posteriori multiple contrasts (all $p s<.05$ ).

There was a main effect of expression $[F(6,266)=25.80$, $\left.M S_{\mathrm{e}}=530.05, p<.0001\right]$, but not of face gender $(F<1)$. Neutral $(M=95.7 \%)$ and happy $(M=98.4 \%)$ expressions were identified more accurately than all the other expressions. Angry $(M=84.7 \%)$ and sad $(M=84.7 \%)$ faces were identified more accurately than fearful $(M=75.1 \%)$ faces, although not more than disgusted $(M=81.9)$ and surprised $(M=81.3 \%)$ faces, which did not differ from fearful faces. There was also a main effect of display time $\left[F(6,1064)=67.68, M S_{\mathrm{e}}=28.70, p<.0001\right]$. Expres- sions were more poorly identified in the 25 -msec display $(M=82.6 \%)$ than in all other conditions, and more poorly in the 50 - and 100 -msec displays $(M=84.6 \%$ and $85.4 \%$, respectively) than in the 250-and 500-msec displays ( $M=$ $88.5 \%$ and $88.8 \%$, respectively).

Nevertheless, all these effects were qualified by a type of expression $\times$ display time interaction $[F(6,1064)=$ $\left.3.26, M S_{\mathrm{e}}=28.70, p<.0001\right]$. To decompose this interaction, the effects of type of expression were examined for each display time condition separately [for the 25-msec display, $F(6,273)=20.77, M S_{\mathrm{e}}=161.89, p<$ .0001 ; for the 50-msec display, $F(6,273)=21.77, M S_{\mathrm{e}}=$ $157.44, p<.0001$; for the 100 -msec display, $F(6,273)=$ $25.22, M S_{\mathrm{e}}=115.19, p<.0001$; for the 250 -msec display, $F(6,273)=20.92, M S_{\mathrm{e}}=109.31, p<.0001$; for the 500-msec display, $F(6,273)=20.30, M S_{\mathrm{e}}=101.81, p<$ $.0001]$. Tukey contrasts $(p<.05)$ showed, first, that for all displays, happy and neutral expressions were identified more accurately than all the other expressions; second, that angry, sad, disgusted, and surprised expressions were identified with comparable accuracy across all displays; and, third, that fearful faces were identified more poorly than sad faces in the 25-msec display, than sad and angry faces in the 50- and 100-msec displays, than all the other faces in the 250-msec display, and than angry faces in the 500-msec display.

\section{GENERAL DISCUSSION}

The major findings of these two studies were consistent and revealed, first, that there were no differences as a function of gender of participant on emotional face

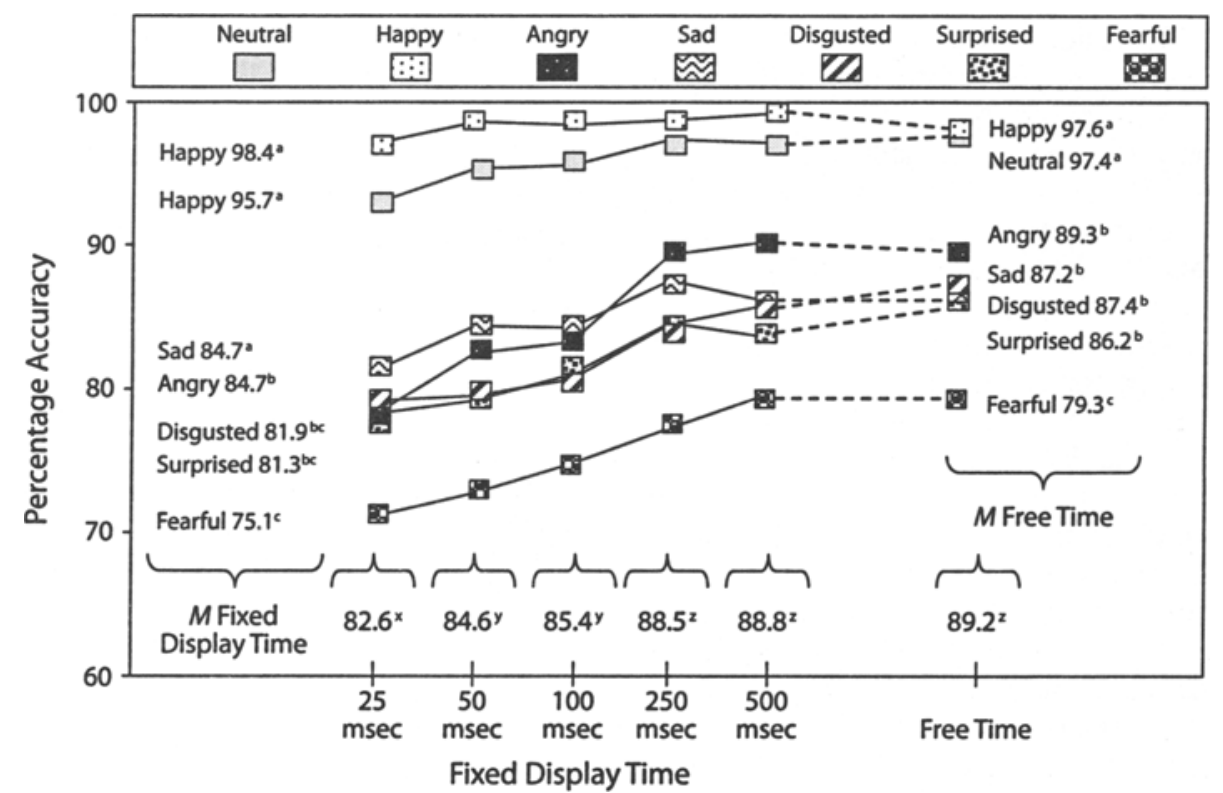

Figure 3. Percentage of participants who correctly judged the intended expression, as a function of display time. For vertical comparisons (left: fixed-display time; right: free-viewing time), means without identical superscript letters $(\mathrm{a}, \mathrm{b}$, or $\mathrm{c}$ ) are significantly different from each other (if mean scores share one superseript letter, they are equivalent). For horizontal comparisons (comparisons across fixed-display and free-viewing conditions), means with different superseript letters $(x, y$, or $z)$ are significantly different from each other. 
identification; also, the effect of model gender (face gender) was weak, with only a tendency for anger to be more likely misperceived as disgust in female than in male faces, and for disgust to be more likely misperceived as anger in male than in female faces. Second, happy and neutral expressions were better identified (both greater accuracy and shorter reaction times) than all the other expressions, whereas fearful expressions were the least likely and the latest to be accurately identified. Identification accuracy and reaction time for angry, sad, disgusted, and surprised expressions were between the two extremes, with relatively small differences among these four expressions. Third, there were systematic errors of misperception among expressions, particularly between anger and disgust (and vice versa) and between surprise and fear (and vice versa), with sadness being misperceived as fear or disgust. Fourth, the identification threshold was particularly low for happy faces. Whereas recognition for all the other expressions improved across increased display durations, the accuracy level for happy faces reached a ceiling level at 50-msec display, which was not further improved by longer displays.

These results are consistent with those of a similar study conducted by Palermo and Coltheart (2004). These authors also found that there were generally no effects or interactions involving participant gender, and relatively few differences as a function of face gender; that recognition rate was highest and naming latencies shortest for happy faces, whereas recognition rate was lowest and naming latencies longest for fearful faces; and that there were misclassifications between surprise and fear, and between anger and disgust. It is important to note that such a comparable pattern emerged in the present study and in the Palermo and Coltheart study even though a different face database was used, as well as different measures to assess accuracy and reaction times (i.e., motor vs. verbal responses). Recognition accuracy was generally higher in our study (except for happy and surprised faces, with no differences between the two studies), especially for the least accurately identified expression-that is, fear (51.7\% vs. $79.3 \%$ accuracy in the free-viewing condition in Palermo \& Colheart, 2004, vs. the present study, respectively). This suggests that the KDEF stimuli are reliable as facial expressions of emotion.

Our data can also be compared with those obtained by Ekman and Friesen (1976) for the PFA, which have often been used in prior research on facial emotion perception. These authors presented norming data for $\mathbf{1 0}$ models for whom all expressions are available. The PFA were shown to participants for $10 \mathrm{sec}$ each. Identification accuracy but not reaction times were collected. In general, if we compare these data with those of the present study, there are small differences in accuracy $(<3 \%$ difference for the expressions of happiness, sadness, and anger; $\leq 6 \%$ difference for surprise, disgust, and neutral), except for fear (a $10.2 \%$ difference, with PFA scores being higher). In both studies, the happy expressions were identified best and fear was identified worst (along with anger, in the Ekman \& Friesen, 1976, data). In general, although the PFA scores for all expressions were very high (above $89 \%$ accuracy), the stimulus sample was limited. With slightly lower recognition accuracy, the KDEF offers a larger stimulus database. In addition, it provides reaction time data, as well as accuracy under a range of restricted viewing conditions, which is useful for estimating relative thresholds for each type of face and expression.

The happy face superiority effect has also been found in other paradigms, such as choice-reaction times (Leppänen, Tenhunen, \& Hietanen, 2003), identification under backwardly masked presentation (Maxwell \& Davidson, 2004), and visual search (Juth, Lundqvist, Karlsson, \& Öhman, 2005) of photographs of real faces. The deficit in the identification of fearful faces has also been reported by some prior research (see Russell, 1994). The happy face advantage and the fearful face disadvantage may be due to happy expressions being more distinctive than other expressions, in the sense of containing fewer overlapping features with other expressions, whereas fearful faces are easily confused with surprised faces (see also Adolphs, 2002).

Nevertheless, the specific contribution of the present study is not so much to determine the differences between categories of facial expressions (or to provide the corresponding explanations) as to provide norms regarding differences between individual exemplars of these categories. For this purpose, we have obtained normative scores for each face stimulus of the KDEF database. Beyond the average values for each expression category, the present study aims to be useful by providing individual values (in response accuracy, RTs, threshold levels, type of errors) for each face and model relative to other faces and models. This is expected to assist researchers in selecting the most appropriate face stimuli when they conduct studies on the processing of emotional faces. Researchers can consult this information in AppendixA.xls (see Archived Materials).

\section{AVAILABILITY}

A CD containing the Karolinska Directed Emotional Faces (Lundqvist et al., 1998), as well as the cropped faces and validation data, is available for purchase from www .facialstimuli.com for $€ 100$. The CD may be used by research institutes for noncommercial purposes only.

\section{AUTHOR NOTE}

This research was supported by Grant SEJ2004-420/PSIC from the Spanish Ministry of Education and Science. The authors have no commercial or financial interest in the KDEF materials (hardware or software) that are referred to in this article. Correspondence concerning this article should be sent to M. G. Calvo, Departamento de Psicologia Cognitiva, Universidad de La Laguna, 38205, Tenerife, Spain (e-mail: mgcalvo@ull.es).

\section{REFERENCES}

ADOLPHS, R. (2002). Recognizing emotion from facial expressions: Psychological and neurological mechanisms. Behavioral \& Cognitive Neuroscience Reviews, 1, 21-62.

Calvo, M. G., \& Esteves, F. (2005). Detection of emotional faces: Low perceptual threshold and wide attentional span. Visual Cognition, 12, 13-27.

Compton, R. (2003). The interface between emotion and attention: A review of evidence from psychology and neuroscience. Behavional \& Cognitive Neuroscience Reviews, 2, 115-129. 
Ekman, P., \& Friesen, W.V. (1976). Pictures of facial affect. Palo Alto, CA: Consulting Psychologists Press.

FaRAH, M. J. (2000). The cognitive neumoscience of vision. Oxford: Blackwell.

Gur, R. C., Sara, R., Hagendoorn, M., Marom, O., Hughett, P., MACY, L., ET AL. (2002). A method for obtaining 3-dimensional facial expressions and its standardization for use in neurocognitive studies. Journal of Neuroscience Methods, 115, 137-143.

JuTh, P., Lundevist, D., KARLsson, A., \& OhmaN, A. (2005). Looking for foes and friends: Perceptual and emotional factors when finding a face in the crowd. Emotion, 5, 379-395.

Leppänen, J., Tenhunen, M., \& Hietanen, J. (2003). Faster choicereaction times to positive than to negative facial expressions. Journal of Psychophysiology, 17, 113-123.

Lundovist, D. Flyxt, A., \& Örman, A. (1998). The Karolinska Directed Emotional Faces-KDEF [CD-ROM]. Department of Clinical Neuroscience, Psychology section, Karolinska Institutet, Stockholm, Sweden.

MAXWELL, J. S., \& DAvidson, R. J. (2004). Unequally masked: Indexing differences in the perceptual salience of "unseen" facial expressions. Cognition \& Emotion, 18, 1009-1026.

MAzURSKI, E. J., \& BoND, N. W. (1993). A new series of slides depicting facial expressions of affect: A comparison with the pictures of the facial affect series. Australian Joumal of Psychology, 45, 41-47.

Morris, J. S., OHMAN, A., \& Dolan, R. J. (1998). Conscious and unconscious emotional learning in the human amygdala. Nature, 393, 467-470.

Palermo, R., \& Coltheart, M. (2004). Photographs of facial expression: Accuracy, response times, and ratings of intensity. Behavior Research Methods, Instruments, \& Computers, 36, 634-638.

Palermo, R., \& Rhodes, G. (2007). Are you always on my mind? A review about how face perception and attention interact. Neuropsychologia, 45, 75-92.

PEsson, L. (2005). To what extent are emotional visual stimuli processed without attention and awareness? Current Opinion in Neurobiology, 15, 1-9.

RUSSELL, J. A. (1994). Is there universal recognition of emotion from facial expression? A review of cross-cultural studies. Psychological Bulletin, 115, 102-141.

SCHNEIDER, W., EschmAN, A., \& ZuccolotTo, A. (2002). E-Prime user's guide. Pittsburgh, PA: Psychology Software Tools.

SChUPP, H. T., Öman, A., JUnghöfer, M., WeIKe, A. I., STOCKBURGER, J., \& HAMM, A. (2004). The facilitated processing of threatening faces: An ERP analysis. Emotion, 2, 189-200.
StENBERG, G., WIKING, S., \& DAHL, M. (1998). Judging words at face value: Interference in a word processing task reveals automatic processing of affective facial expressions. Cognition \& Emotion, 12, 755-782.

TotTenham, N., Borscheid, A., Ellertsen, K., Marcus, D. J., \& Nelson, C. A. (2002). Categorization of facial expressions in children and adults: Establishing a larger stimulus set [Abstract]. Journal of Cognitive Neuroscience, 14(Suppl.), S74.

VuILleUMier, P., \& PourToIs, G. (2007). Distributed and interactive brain mechanisms during emotion face perception: Evidence from functional neuroimaging. Neuropsychologia, 45, 174-194.

Watson, T. (2001). Perceptual categorization of anti-expressions. Unpublished honors thesis, Macquarie University, Sydney, Australia.

Winliams, M. A., Moss, S. A., Bradshaw, J. L., \& Mattingley, J. B. (2005). Look at me, I'm smiling: Visual search for threatening and nonthreatening facial expressions. Visual Cognition, 12, 29-50.

\section{ARCHIVED MATERIALS}

The following materials associated with this article may be accessed through the Psychonomic Society's Norms, Stimuli, and Data archive, www.psychonomic.org/archive/.

To access these files, search the archive for this article using the journal (Behavior Research Methods), the first author's name (Calvo), and the publication year (2008).

FLLE: Calvo-BRM-2008.zip

DESCRIPTION: The compressed archive file contains three files:

CalvoEtAl-BRM-APP-A.xls, containing the norming data developed by Calvo and Lundqvist as a tab-delimited text file generated by Excel for Windows XP. Each row represents one of the 280 faces rated. The 59 columns contain identifying information of the variables.

CalvoEtAl-BRM-APP-B.pdf, containing a full description of the content of AppendixA.xls, including extended definitions of the columns of the document.

CalvoEtAl-BRM-APP-C.pdf, containing a list of publications that have used the KDEF database, which can also be useful information for researchers.

AUTHORs' E-MAIl ADDRESSEs: mgcalvo@ull.es, daniel.lundqvist@ki.se

(Manuscript received November 15, 2006; revision accepted for publication March 13, 2007.) 